\title{
Numerical Simulation of Multi-chamber Piston Compressed Ignition Engine
}

\author{
${ }^{1}$ Manoj Kumar Sharma, ${ }^{2}$ K.P.Chandraiah, ${ }^{3}$ Gohil Priyank \\ ${ }^{I}$ M.Tech. Scholar, TIEIT Bhopal (M.P.) India \\ ${ }^{2}$ Asst.Professor, SIET Tumkur (Karnataka) India \\ ${ }^{3}$ M.Tech. Student, TIEIT ,Bhopal (India)
}

\begin{abstract}
Due to increase in the demand and the scarcity in the availability of the fossil fuels, it is essential to search for the effective burning of fuel in the existing system. In diesel engines the fluid motion within the engine cylinder is one of the major factors that controls the fuel-air mixing, combustion processes and also has a significant effect on heat transfer. Both the bulk gas motion and the turbulence characteristics of the flow are important for the performance of C.I engine. The present work relates to the modification of the C.I engine design for inducing turbulence by squish and tumble flows to improve the combustibility of the combustible mixture. This modification includes the formation of multi-chambers on the piston crown. It consists of three small chambers at $120^{\circ}$ apart. The C.I engine with multi-chamber piston at motoring condition has been analyzed through CFD using FLUENT software and the results obtained are compared with the base C.I engine. The tumble ratio is increased by $35 \%$ and squish velocity is increased by $31 \%$ with the modified engine in comparison with the base engine.
\end{abstract}

Key words: Flow patterns, C.I Engine Cycle, Governing equation in CFD, Modeling and Meshing, Geometric Model Creation, Geometry Decomposition-Mesh generation, Mesh motion scheme.

\section{Introduction}

The in-cylinder flows of C.I engine is an important parameter for researchers \& scientists. It is due to the fact that the flow structure generated by intake flows is related closely to the design and performance of the C.I engine. The production of high turbulence intensity is one of the most important factors for stabilizing the combustion process. In general two types of vertices are utilized in order to generate and preserve the turbulence flows efficiently. These vertices are usually known as swirl and tumble flows, which are organized rotations in the horizontal and vertical plane of the engine cylinder respectively. It contributes in improving the engine performance by accelerating mixing of fuel and induced air.

A few experimental works in visualizing in-cylinder flows have been conducted to measure velocity fields by using hot wire anemometry or laser Doppler velocimetry. However, it is a really hard task to perform it because the measurements of in-cylinder flows in the reciprocating engine are characterized by highly complex three-dimensionality, turbulence \& unsteadiness. A numerical approach could thus be an alternative because of the capability of CFD which has been developed for in-cylinder flow predictions in recent years. The CFD package FLUENT is most efficient for in-cylinder flow analysis. The CFD models should cover the specific problems related to the turbulent flow, high Reynolds number, compressible flow and the complex geometry.

The most usual numerical method in Computational Fluid Dynamics (CFD) is finite volume method (FVM). In this investigation, an important fluid flow pattern in CFD simulations, namely, Tumble motion typical in automotive engines is considered and RNG k-e turbulence model were used. The air flow in a twovalve engine cylinder during 720 degree of crank angle

Recently simulation results by CFD codes are used in the development and optimization of new engines by automotive industry.

\section{Flow Patterns}

Swirl is defined as organized rotation of the charge about the cylinder axis. Swirl is created by bringing the intake flow into the cylinder with an initial angular momentum. Swirl is used in diesel engine to promote more rapid mixing between the inducted air charge and the injected fuel. Swirl is also used to speed up the combustion process. The swirl motion inside the engine cylinder is shown in fig 1.

Tumble is a rotational motion about a circumferential axis near the edge of the clearance volume in the cylinder head, which is caused by squishing of the in-cylinder volume as piston reaches near TDC. These values are calculated by the effective angular speed of in-cylinder air motion divided by the engine speed. The effective angular speed is the ratio of the angular momentum to the angular inertia of moment. The tumble motion inside the engine cylinder is shown in fig 1. 


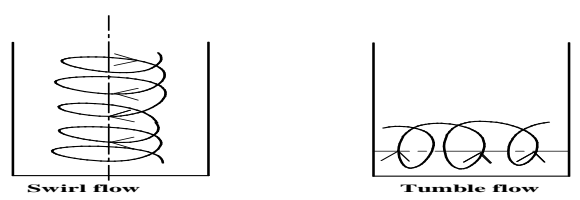

Fig 1 Swirl and Tumble flow in a cylinder.

Squish is the radically inward or transverse gas motion that occurs toward the end of the compression stroke Squish-generated gas motion results from using compact combustion chamber geometry. Fig 2 shows piston motion generates squish in bowl-in-piston direct-injection diesel combustion chamber.

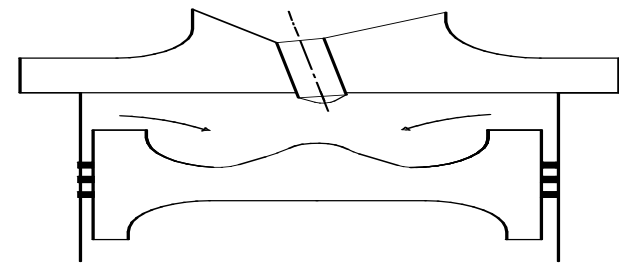

Fig 2 squish generation in CI engine.

\section{Objective Of The Study}

To perform modeling, meshing and flow analysis of multi- chamber piston C.I engine and base engine in CFD

$>$ The variation of various properties i.e. pressure, temperature, swirl ratio, tumble ratio are studied at different crank angles

$>$ To reduce the cost and time of experiments by predicting the performance of C.I engine by analyzing using CFD.

\section{Overview Of Diesel Engine}

A Diesel engine is an internal combustion engine which operates using the Diesel cycle. German engineer Rudolf Diesel invented it in 1892. He received a patent for it on February 23, 1893.

In Diesel Engine the fuel ignites upon being injected into the highly compressed air in the combustion chamber.

\section{How Diesel Engine Works}

In mechanical terms, the internal construction of a Diesel engine is similar to its gasoline counterpartcomponents such as pistons, connecting rods and a crankshaft are present in both. Like a gasoline engine, a Diesel engine may operate on a four-stroke cycle (similar to the gasoline unit's Otto cycle), or a two-stroke cycle. In both cases, the principal differences lie in the handling of air and fuel, and the method of ignition.

A Diesel engine relies upon compression ignition to burn its fuel, instead of the spark plug used in a gasoline engine. If air is compressed to a high degree, its temperature will increase to a point where fuel will burn upon contact. This principle is used in both four-stroke and two-stroke Diesel engines to produce power. A Diesel's compression ratio is usually between 16:1 and 25:1. This extremely high level of compression causes the air temperature to increase to 700 to 900 degrees Celsius (1300 to 1650 degrees Fahrenheit). Fuel oil is injected into the cylinder at high pressure, causing the fuel charge to be atomized. Owing to the high air temperature in the cylinder, ignition instantly occurs, causing a rapid and considerable increase in cylinder temperature and pressure.

\section{P-V \& T-S Diagram Of C.I Engine Cycle}

This cycle was introduced by Rudolph Diesel, which is applicable for slow speed compression ignition engines or diesel engines. Heat addition takes place at constant pressure and hence the cycle is also known as constant pressure cycle. This cycle consists of two reversible adiabatic, one constant pressure process and one constant volume process. Heat rejection takes place during constant volume process. Different processes are shown on the P-V diagram in fig.3. 1-2 adiabatic compression process, 2-3 constant pressure process, 3-4 adiabatic expansion process, 4-1 drop in pressure at constant volume.

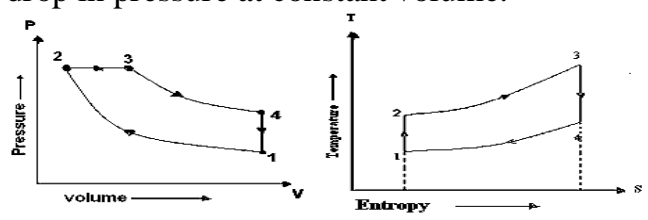

Fig 3 P-V and T-S diagram 


\section{Different Types Of Combustion Chambers In Ci Engine}
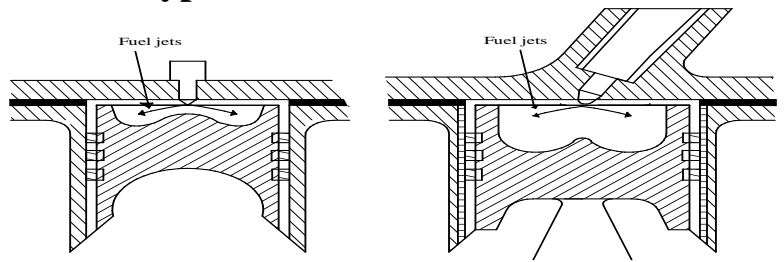

Fig. 4 Quiescent chamber with multi hole nozzle. B. bowl-in-piston chamber with swirl and multi hole nozzle.

In the largest-size engines, where mixing rate requirements are least stringent, quiescent direct injection system shown in fig 4A are used. The momentum and energy of the injected fuel jets are sufficient to achieve adequate fuel distribution and rates of mixing with the air. Additional organized air motion is not required. The combustion chamber shape is usually a shallow bowl in the crown of the piston, and a central multi-hole injector is used. As engine size decreases, increasing amounts of air swirl are used to achieve faster fuel-air mixing rates. Air swirl is generated by suitable design of the inlet port, the swirl rates can be increased as the piston approaches TDC by forcing the air toward the cylinder axis, into a bowl-in-piston type of combustion chamber shown in fig $3.2 \mathrm{~B}^{[8]}$.

\section{Introduction To CFD}

Fluid dynamics deals with the dynamic behavior of fluids and its mathematical interpretation is called as Computational Fluid Dynamics. Fluid dynamics is governed by sets of partial differential equations, which in most cases are difficult to obtain analytical solution. CFD is a computational technology that enables the study of dynamics of things that flow.

The Physical aspects of any fluid flow are governed by three fundamental principles: Mass is conserved; Newton's second law and Energy is conserved. These fundamental principles can be expressed in terms of mathematical equations. Computational Fluid Dynamics (CFD) is the science of determining a numerical solution to the governing equations of fluid flow.

Computational Fluid Dynamics (CFD) thus provides a qualitative (and sometimes even quantitative) prediction of fluid flows by means of Mathematical modeling (partial differential equations) Numerical methods (discretisation and solution techniques) Software tools (solvers, pre- and post processing utilities) CFD enables scientists and engineers to perform 'numerical experiments' (i.e. Computer simulations) in a 'virtual flow laboratory' for real experiment CFD simulation.

i. Results can be obtained from the graphic display and report in FLUENT. Results can be displayed in terms of contour, velocity vector, particle track and path line. Any calculation required can be performed in FLUENT also.

ii. Finally, the results and all the data can be saved for future references by writing the files.

\section{Governing Equations In CFD}

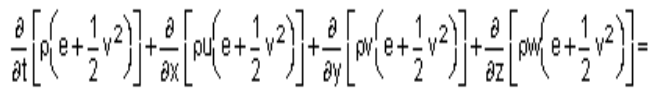

$$
\begin{aligned}
& h\left(\frac{\partial^{2} T}{\partial x^{2}}+\frac{\partial^{2} T}{\partial y^{2}}+\frac{\partial^{2} T}{\partial z^{2}}\right)-\left(u \frac{\partial p}{\partial x}+v \frac{\partial p}{\partial y}+W \frac{\partial p}{\partial z}\right)
\end{aligned}
$$

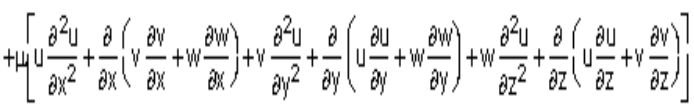

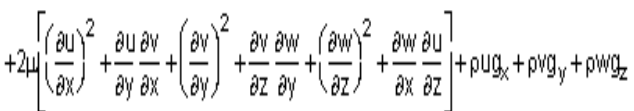

\section{(a) Continuity Equation}

Continuity equations often can be expressed in either integral or differential form as shown below.

$$
\int_{c s} \rho V d A+\frac{\partial}{\partial t} \int_{c v} \rho d A=0
$$

This is a statement of the principle of mass conservation for a steady, one-dimensional flow, with one inlet and one outlet.

$$
\nabla(\rho V)+\frac{\partial \rho}{\partial t}=0
$$




$$
\text { Where, } \nabla=\frac{\partial}{\partial x} \hat{i}+\frac{\partial}{\partial y} \hat{j}+\frac{\partial}{\partial z} \hat{k}
$$

(b) $\quad \frac{\partial \rho}{\partial \mathrm{t}}+\frac{\partial(\rho \mathrm{u})}{\partial \mathrm{x}}+\frac{\partial(\rho \mathrm{v})}{\partial \mathrm{y}}+\frac{\partial(\rho \mathrm{w})}{\partial z}=0$

\section{Momentum (Navier Stokes) Equations}

The momentum equation is a statement of Newton's Second Law and relates the sum of the forces acting on an element of fluid to its acceleration or rate of change of momentum. The Newton's second law of motion $\mathrm{F}=$ ma forms the basis of the momentum equation.

$$
\begin{aligned}
& \rho\left[\frac{\partial u}{\partial t}+u \frac{\partial u}{\partial x}+v \frac{\partial u}{\partial y}+w \frac{\partial u}{\partial z}\right]=\rho g_{x}-\frac{\partial p}{\partial x}+t \\
& \rho\left[\frac{\partial v}{\partial t}+u \frac{\partial v}{\partial x}+v \frac{\partial v}{\partial y}+w \frac{\partial v}{\partial z}\right]=\rho g_{y}-\frac{\partial p}{\partial y}+\mu \\
& \rho\left[\frac{\partial w}{\partial t}+u \frac{\partial w}{\partial x}+v \frac{\partial w}{\partial y}+w \frac{\partial w}{\partial z}\right]=\rho g_{z}-\frac{\partial p}{\partial z}+
\end{aligned}
$$

\section{(c) Energy Equation}

In situations where the fluid may be treated as incompressible and temperature differences are small, the continuity and momentum equations are sufficient to specify the velocities and pressure (that is, four equations (Continuity +3 -Momentum) and four unknown quantities $[\mathrm{u}, \mathrm{v}, \mathrm{w}$ and $\mathrm{p}]$ ). If the flow is compressible ( $\rho$ is not constant), or if heat flux occurs (temperature not constant), at least one additional equation is required. In some of these instances, the Energy equation may be used.

\section{The Advantages Of CFD}

These are the compelling reasons to use CFD :

Insight: There are many devices and systems that are very difficult to prototype. Often, CFD analysis shows parts of the system or phenomena happening within the system that would not otherwise be visible through any other means. CFD gives a means of visualizing and enhanced understanding of the designs.

Foresight: Because CFD is a tool for predicting what will happen under a given set of circumstances, In a short time, CFD can predict design performance, and hence number of variants can be tested until optimal result is found. All is done before physical prototyping and testing. This foresight made available to helps engineers and designers to analyze shorter design cycles and save the time and money.

\section{Applications Of CFD}

when fluids flow with the complications of:

- Simultaneous flow of heat,

- Mass transfer (perspiration, dissolution),

- Phase change (melting, freezing, boiling),

- Chemical reaction (combustion, rusting),

- Mechanical movement ( pistons, fans, rudders)

- Stresses and displacement of immersed or surrounding solids.

How fluids will flow and their quantitative effects on the solids, which can assist:

1. Building-service engineers and architects to provide comfortable and safe human environments

2. Power-plant designers to attain maximum efficiency, and reduce release of pollutants Chemical engineers to maximize the yields of their reactors and processing equipment

\section{Limitations Of CFD}

CFD-based predictions are never $100 \%$ - reliable because of the following reason:

1. The input data may involve.

2. The available computer power may be too small for high numerical accuracy.

3. For laminar flows rather than turbulent

4. For single-phase flows rather than multi-phase flows

5. For chemically-inert rather than chemically-reactive materials

6. For single chemical reactions rather than multiple ones complex composition

\section{Modeling And Meshing}

In the present work modeling has been done by using CATIA software, the modeled geometry is meshed using HYPERMESH tool. Fig 5. shows the two dimensional view of CI engine with multi -chamber piston 

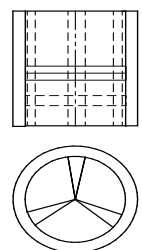

Fig 5 Two dimensional view multi -chamber piston of CI engine

\section{Engin Specifications}

Table 1 Engine specification

\begin{tabular}{|c|c|c|}
\hline $\begin{array}{l}\text { SL } \\
\text { NO }\end{array}$ & $\begin{array}{c}\text { ENGINE } \\
\text { PARAMETERS }\end{array}$ & SPECIFICATION \\
\hline 01 & Engine Type & TV1(kirloskar,four stroke) \\
\hline 02 & Number of Cylinders & Single cylinder \\
\hline 03 & Number of strokes & Four-stroke \\
\hline 04 & Rated power & $5.2 \mathrm{KW}(7 \mathrm{HP}) @ 1500 \mathrm{rpm}$ \\
\hline 05 & Bore & 87.5 \\
\hline 06 & Stroke & $110 \mathrm{~mm}$ \\
\hline 07 & Cubic Capacity & $661 \mathrm{cc}$ \\
\hline 08 & Compression ratio & $17.5: 1$ \\
\hline 09 & Rated speed & $1500 \mathrm{rpm}$ \\
\hline 10 & Dynamometer & $\begin{array}{c}\text { Eddy current dynamometer, } \\
\text { make SAJ }\end{array}$ \\
\hline 11 & Type of cooling & Water cooling \\
\hline 12 & $\begin{array}{l}\text { Fuel injection } \\
\text { pressure }\end{array}$ & $190 \mathrm{Bar}$ \\
\hline 13 & Fuel & Diesel \\
\hline
\end{tabular}

\section{Geometric Model Creation}

The geometry of computational domain from the analysis point of view can be created by top-down or bottom-up methods. Top-down refers to an approach where the computational domain is created by performing logical operations on primitive shapes. Geometries are created using the same pre-processor software that is used to create the grid, or created using other programs (e.g. CAD, graphics). Geometry files are imported into HM to create computational domain. The Extracted fluid domain of CI engine as shown in Fig.5.1

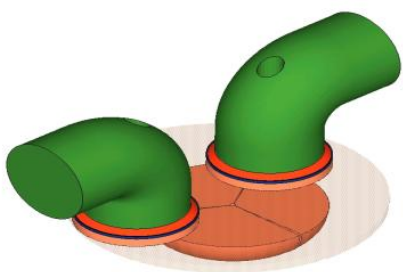

Fig.6 CFD Model of computational domain

The fig 7 shows the CFD Model computational domain which is done by using CATIA and it is then extracted for meshing and analysis.

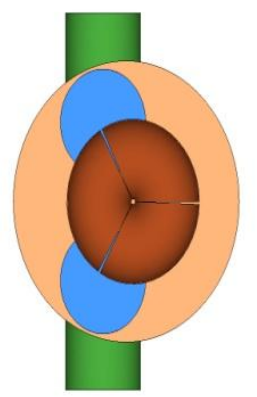




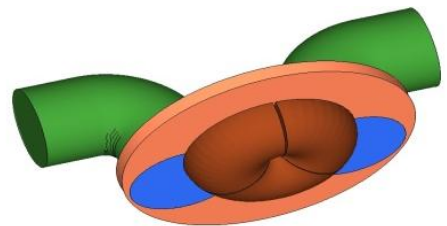

Fig.7 Isometric View of Computational Domain

The fig 7 shows the isometric view of computational domain used in the present work. It clearly shows the multi-chamber piston in sectional views and also when piston is at TDC, it is then meshed by HYPERMESH using appropriate elements or cells.

\section{Geometry Decomposition-Mesh Generation}

Two approaches are employed in Fluent6.3 to solve in-cylinder problems, namely, hybrid approach and layering approach. While the hybrid approach is used for engines with canted valves. Fluent consist of three stages:

$>$ The first stage is to decompose the geometry into different zones and mesh them properly to apply different mesh motion strategies to different regions in a single simulation.

$>$ The second stage is to set up the engine case inside Fluent with the help of a setup journal.

$>$ The third stage is to perform a transient in-cylinder simulation.

The decomposition process is shown in Fig.9.

For this project a hybrid mesh is generated using Hyper Mesh. Hybrid mesh is the combination of Tetrahedron and hexahedron elements

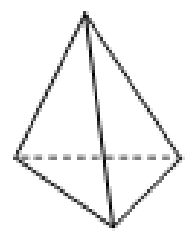

Tetrahedron

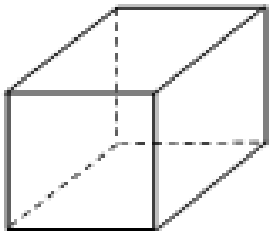

Hexahedron

Fig.8 Geometry decomposition
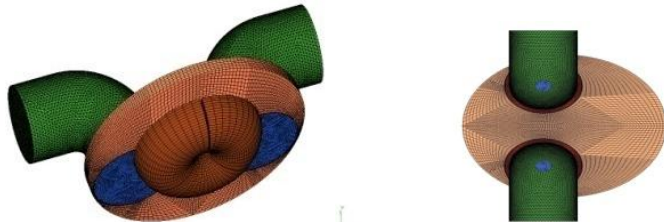

Fig.9 Close view of CFD domain mesh

\section{Mesh Motion Scheme}

Mesh motion scheme indicates the movement of cells or elements without failure after complete meshing, in order to achieve this computational geometry is made simple to suit for cooper mesh technique. Following are the assumptions incurred on the present analysis:

(a) Flow is Transient and incompressible (b) Flow is Turbulent (c) Segregated solver The decomposition and zone name matching explained in Fig.6.1 andFig.6.2 contains a sketch of the decomposition and the corresponding zone names. At the inlet, pressure boundary condition is applied. The engines walls are defined as stationary no slip walls.

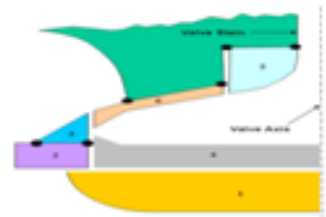

Fig.10 Fluid zone names and mesh requirement

\section{In-Cylinder Setup}

The In-Cylinder setup shows the setup panel on computer monitor in which the required parameters are incorporated which are suited for the present work. Setting up of an engine parameters is very important because the results like peak pressure, temperature is mainly depend on the this step, because here all the data has been incorporated, this data has to be given by the solver and it mainly indicates the specifications of the engine used 
to run. There the starting crank angle, stroke length, engine speed etc... are incorporated to the solver according to our requirements.
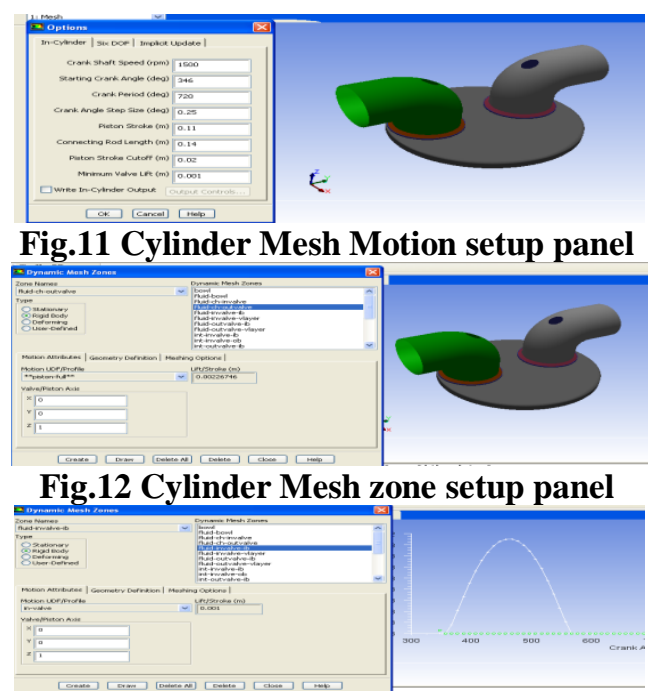

Fig.13 In cylinder zone setup panel

Fig 11 shows the setup panel in which engine parameters which are required for the present work are imputed. Fig 12 shows the parameters which are selected in order to obtain piston motion. Fig 13 shows the selected parameters for valve motion.

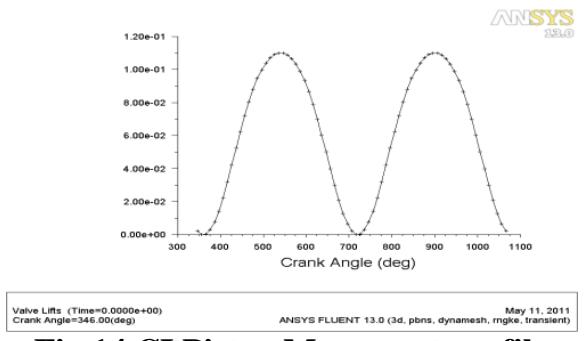

Fig.14 CI Piston Movement profile

The profile of CI Engine Piston movement is as shown in the figure 14, from figure we can easily see the movement of the piston. If any variations are required in the movement this figure helps in predicting the movement of the piston before the start of analysis. Where the piston movement starts from an angle of $340^{\circ}$ and end at an angle of $1070^{\circ}$. In order to complete one cycle of operation.

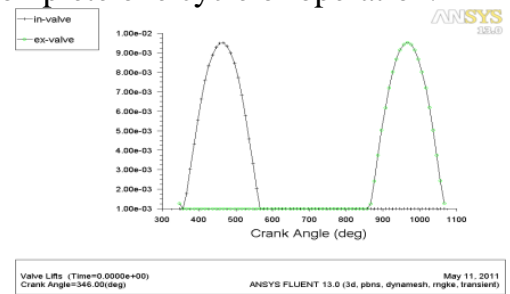

Fig.15 CI in-valve profile

The profile of CI Engine Valve movement is as shown in the figure 15, the inlet valve starts opening at an angle of $340^{\circ}$ and it will remain opened till it reaches $570^{\circ}$ and it closes till the start of next cycle. The exhaust valve will be closed during the operations. The power strokes opens at an angle of $870^{\circ}$ and will close after the burnt gases to be exhausted.

\section{Results And Discussions}

In CFD analysis for both engines base and modified engine. The peak pressure, tumble ratio and squish velocity are discussed at different crank angles. The CFD output in terms of contours of pressure, temperature and velocity are obtained as soon as the runs completes. It is very difficult to compare the results with those contours; hence these contours are used to draw the Graphs.

In the present work, the detailed numerical study has been carried out to investigate the effect of combustion chamber geometry on the fluid flow field characteristics of a 4-stroke C.I engine under motoring condition at a speed of 1500rpm.

In cylinder set up i.e., the physical phenomena like speed of the engine, starting and ending angle of crank, stroke length etc adopted in the present work shown in table 2. 


\begin{tabular}{|l|l|l|}
\hline S1 No & Parameters & Values \\
\hline 1 & Crank shaft speed (rpm) & 1500 \\
\hline 2 & Starting Crank angle (deg) & 346 \\
\hline 3 & Crank period (deg) & 720 \\
\hline 4 & Crank Angle Step Size (deg) & 0.25 \\
\hline 5 & Piston Stroke (m) & 0.11 \\
\hline
\end{tabular}

Table 2 gives the in cylinder setup adopted for the present work.

Then the setup run has been done while the software solves all the three transport equations to obtain the contours of velocity, pressure and temperature.

\section{Conclusion}

To improve the air flow characteristics of 4 stroke diesel engine, the base engine piston was modified with multi-chamber on the piston crown. The investigation and analysis of air flow characteristics during suction and compression strokes were carried out using CFD code of Fluent 6.3 with dynamic mesh. After the analysis the following results were drawn:

1. The peak tumble ratio of the modified engine was found to be 0.88 in comparison with the base engine of 0.65 . Thus an improvement of $35 \%$ of tumble ratio is achieved.

2. The peak tumble ratio in modified engine shifts towards TDC by $10^{\circ}$ compared to the base engine.

3. The squish velocity of the modified engine was increased by $31 \%$ in comparison with the base engine.

4. The maximum cylinder pressure in the modified engine was found to be 55 bar in comparison with the base engine of 38 bar. Thus an improvement of $45 \%$ of pressure is achieved.

5. The peak pressure in modified engine shifts towards TDC by $10^{\circ}$ compared with the base engine.

6. It is clear from the study that the modified piston has the capability of producing higher tumble and squish which will improve the combustibility of combustible mixture during combustion.

\section{Scope Of Future Work}

The present work has been carried out to improve the induced turbulence of 4-S diesel engine by considering the effects of tumble and squish under motoring condition only. The following proposals are made for the future work:

$>$ Detailed research can be made on the combustion \& emission characteristics with the modified combustion chamber.

$>$ By using alternate fuel like bio-diesel for this modified engine piston because of increased turbulence.

$>$ The performance characteristics can be studied for different compression ratio, Intake and exhaust manifolds geometry.

\section{References}

[1]. Code coupling to enhance cfd analysis of i.c. Engines by Gino Bella*, Rossella Rotondi*, Gabriel Defranco $\dagger$ y Norberto Nigro $†$, Mecánica Computational Vol. XXIIM. B. Rosales, V. H. Cortínez y D. V. Bambill (Editores)Bahía Blanca, Argentina, November 2003, p.no. 26-40

[2]. Flow field development in a direct injection diesel engine with different manifolds by Benny Paul1*, V. Ganesan2, International Journal of Engineering, Science and Technology Vol. 2, No. 1, 2010, pp. 80-91

[3]. Computational Analysis Of Egr Mixing Inside The Intake System \& Experimental Investigation On Diesel Engine For Lcv by S Karthikeyan R. Hariganesh, M.sathyanadan S. Krishnan, p. Vadivel, D vamsidhar, International Journal of Engineering Science and Technology (IJEST) Vol. 3 No. 3 Mar 2011 p.no.2350-2357.

[4]. Turbulence and Heat Transfer Analysis of Intake and Compression Stroke in Automotive 4-stroke Direct Injection Engine by Algerian Journal of Applied Fluid Mechanics | Vol 1 | 2007 p.no.37-50.

[5]. Automatic Mesh Motion, Topological Changes and Innovative Mesh Setup for I.C.E. CFD Simulations by T. Lucchini, G. D’Errico

[6]. In An Engine Valve Lift Visualization And Simulation Performance Using Cfd by Semin, Rosli Abu Bakar, Abdul Rahim Ismail and Ismail Ali, Conference on Applications and Design in Mechanical Engineering, 25-26 October 2007, Kangar, Perlis, Malaysia.

[7]. Diesel Engine Simulation with Tabulation of Dynamic Adaptive Chemistry and Adaptive Local Mesh Refinement by G. D'Errico*, D. Ettorre*, T. Lucchini*, F. Contino ${ }^{\circ}$

[8]. Heywood JB. Fluid motion within the cylinder of internal combustion engines. The 1986 Freeman Scholar Lecture.J Fluids Eng $1986 ; 109(1987)$

[9]. F. Payri, J. Benajes, X. Margot, A. Gil "CFD Modeling of the in-Cylinder Flow in Direct-Injection Diesel Engines" Computers \& Fluids 33 (2004) 995-1021.

[10]. A.R. Noorpoor, "Flow Simulation in Engine Cylinder with Spring Mesh" American Journal of Applied Sciences 5 (10): 1336-1343, 2008 ISSN 1546-9239, 2008 Science Publications. 